\title{
Filigrane
}

Écoutes psychothérapiques

\section{Nada ou le féminin échoué}

\section{Suzanne Tremblay}

Volume 17, numéro 2, automne 2008

L’avenir du clinicien II

URI : https://id.erudit.org/iderudit/019426ar

DOI : https://doi.org/10.7202/019426ar

Aller au sommaire du numéro

Éditeur(s)

Revue Santé mentale au Québec

ISSN

1192-1412 (imprimé)

1911-4656 (numérique)

Découvrir la revue

Citer cet article

Tremblay, S. (2008). Nada ou le féminin échoué. Filigrane, 17(2), 164-173.

https://doi.org/10.7202/019426ar

\section{Résumé de l'article}

À travers un récit clinique, l'auteure dresse le portrait d'une des vicissitudes du féminin, le repli dans le vide, la dormance, pour éviter la douleur liée à l'assomption des pulsions et à la perte. d'utilisation que vous pouvez consulter en ligne.

https://apropos.erudit.org/fr/usagers/politique-dutilisation/ 


\title{
Nada ou le féminin échoué 1
}

\author{
suzanne tremblay \\ À travers un récit clinique, l'auteure dresse le portrait d'une des vicissitudes du \\ féminin, le repli dans le vide, la dormance, pour éviter la douleur liée à l'assomption \\ des pulsions et à la perte.
}

«Ce vide est celui qui s'installe entre un regard et le visage qui ne répond pas »

(P. Fedida)

\section{Liminaire}

Toute ressemblance avec des personnes connues n'est que pure coüncidence. Ainsi s'énonce la mise en garde du romancier ou du cinéaste, mise en garde souvent bien inutile puisqu'au contraire, elle nous porte à nous interroger sur lesdites ressemblances. Sans user de la même mise en garde, puisque le matériel que je vous présente s'inspire d'une rencontre qui a vraiment eu lieu, je tiens néanmoins à préciser que c'est ma fiction que je vous présente, puisqu'il ne peut, de toute manière, en être autrement. Nous n'écrivons sur nos patients qu'à partir d'une déroute, de quelque chose en nous qui est touché et qui a besoin de s'élaborer. C'est vrai dans le cas présent. À mesure que le processus avançait, j'avais le sentiment de plus en plus prégnant d'être prise dans une force d'inertie, d'être aspirée par elle. Allions-nous, Nada et moi, vers un non-lieu thérapeutique?

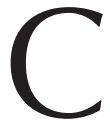

ela ferait bientôt six ans que je voyais Nada, au début une fois puis deux fois par semaine. Six longues années où, bien qu'elle fût plus animée et plus spontanée pendant les séances, rien ne changeait dans sa vie. À part ces deux années où elle retournera compléter, à temps partiel, ce baccalauréat qu'elle avait échoué. Puis, sa vie s'immobilisera de nouveau. Elle sera de plus en plus restreinte, à l'usine, à sa chambre, et à nos séances.

Plus le temps passait, plus je sentais que je faisais désormais partie de sa routine, que je n'étais qu'un rouage de plus dans la mécanique de sa vie. Par moments, l'atmosphère de stagnation, le sentiment d'impuissance rognaient le peu d'espoir qui me restait. Plus d'une fois, j'ai douté du bien-fondé d'avoir entrepris cette cure. Il m'arrivait de parler plus que de coutume, à sa demande. Il y avait tant de choses qu'elle avait besoin que je lui explique. J'avais alors l'impression d'être pour elle la mère qui introduit le monde à son enfant. À d'autres moments, il me fallait parler pour m'animer, tout simplement.

C'est alors que le besoin d'écrire se fit plus impératif. 
Nada ne fait rien, ne désire rien, n'aspire à rien. Depuis son échec en dernière année de baccalauréat, elle a pris le premier emploi qu'on lui a proposé, un travail à la chaîne dans une usine. Elle déteste ce travail, mais il lui convient. «Je peux le faire sans penser, sans rien », me dit-elle. Chaque fois, en enfilant l'uniforme vert, le même pour tous, c'est comme si elle se dépouillait d'elle-même, de son identité. Dès lors, elle n'est plus qu'un rouage de la machine.

«Je n'ai plus de corps, je ne suis qu'une automate, une mécanique. Interchangeable. »

Et lorsqu'elle ne travaille pas, elle se réfugie dans sa chambre, celle-là même qui l'a vue naître et qu'elle n'a jamais quittée. Elle dort. Le plus souvent, d'un sommeil sans rêves. Dormir, pour oublier, pour s'absenter d'elle-même.

Quitter son emploi ou la maison familiale pour se lancer dans la vie, comme elle dit, la terrifie. Dans un rêve qu'elle a fait, il y a quelques mois, elle se tient en équilibre sur une plate-forme inclinée au milieu de l'espace. Entourant la plateforme, sur trois côtés, une rampe de métal - comme celles qu'il y a à son travail. Autour, rien. Que le noir et le vide dans lequel elle risque de tomber si elle lâche prise.

Être sans pensée, sans désir. Une automate. C'est ce que souhaite Nada. «Mourir sans mourir», comme elle me le dit.

Jeune femme au début de la trentaine, plutôt jolie, avec ce qu'elle appelle ellemême un baby face, Nada était venue me consulter pour ce qu'elle appelait ses «tensions », des sensations douloureuses qu'elle éprouvait dans la région vaginale. Ces sensations la troublaient. Plus d'une fois, elles étaient apparues en présence d'une femme. Cela signifiait-il qu'elle était homosexuelle? Si tel était le cas, valait mieux mourir. Elle m'avoua sa fascination pour le corps de la femme, les seins en particulier. Elle se rappelait un tableau ornant la chambre de ses parents lorsqu'elle était enfant, une mère allaitant son bébé, le sein dénudé, du trouble qu'il faisait naître en elle. Cette vision du corps nu de la mère, porteur d'une jouissance innommable, suscitait en elle un désir qui la foudroyait.

Pourtant cette mère, Nada l'avait toujours vécue comme froide, distante, sans gestes tendres. Elle aurait tant aimé la connaître, connaître la jeune fille qu'elle était mais les questions restaient sans réponses. Pourquoi ses parents avaient-ils attendu des années avant d'adopter son frère aîné ? Pourquoi était-elle née dix mois seulement après cette adoption? Elle, l'enfant de chair et de sang, mais retirée du ventre de sa mère par des mains toutes chirurgicales. «Pas même un vrai accouchement», m'avait-elle dit. Elle détestait ce frère qui était, selon elle, l'enfant choisi, adulé par la mère. Alors qu'elle... ? Qu'était-elle sinon le fruit du sexe, de la honte, preuve funeste et vivante de tout de ce qui faisait horreur à sa mère. Pour l'avoir elle, il avait bien fallu «le faire».

Du sexe, on ne parlait pas chez elle. Pour sa mère, les hommes n'étaient que des animaux qui ne pensaient qu'à «ça». Elle avait failli à investir Nada dans son identité de fille et de femme. À ses yeux, elle était restée une enfant. Ni fille, ni garçon. Du genre neutre. Sans sexe. Le corps de la femme, c'est à travers sa 
poupée Barbie que Nada avait pu se le représenter mais en secret, cette poupée évoquant pour la mère la sexualité scandaleuse, les femmes dévergondées, les salopes. La haine de la mère pour son mari (et pour les hommes) emprisonnait Nada dans un lien mortifère. Dès lors, ce couple où il n'y avait pas d'amants n'était pas un couple pour la fille; il n'existait ni pour la mère pour qui les hommes étaient des bêtes ni pour le père qui abdiquait et se laissait exclure.

À ce jour, Nada n'avait eu aucune vie amoureuse. Une amitié avec le copain d'une amie, laquelle avait pris fin brusquement après un rapprochement sexuel, quelques attouchements sans plus. Celui qu'elle appelait son ex-grand ami l'avait quittée le lendemain sans explications. Elle en avait été effondrée pendant des mois. Depuis, les hommes étaient devenus pour elle les prédateurs contre lesquels sa mère l'avait mise en garde.

Elle se voudrait sans sexe. Un lapsus fait dans la première année d'analyse donne la pleine mesure de ce désir. Voulant dire «abstraction», elle avait dit «ablation» de mon sexe. S'amputer de son sexe, c'est ce qu'elle espérait au fond. Que l'analyse la débarrasse de ce qu'elle appelait ses «tensions » qu'elle éprouvait comme des douleurs inquiétantes.

Dans la mesure où elle ne peut avoir de corps à elle, l'image du corps de l'autre femme, de par l'idéalisation dont il est l'objet, s'érotise. Elle devient, éblouissante, captivante, «ravisseuse », pour reprendre un thème cher à Marguerite Duras. Dans Le ravissement de Lol $\mathrm{V}$. Stein, celle-ci décrit très bien cet état d'absence à soimême, cette préférence pour n'avoir pas de corps érotique à soi. «Ce miroir qui ne reflétait rien et devant lequel elle devait ressentir l'éviction soudaine de sa personne. » $(1964,124)$. Lol désire le remplacement, l'effacement de son corps par celui de l'autre femme. «Ce corps infirme de l'autre», « où le mettre exactement, pour qu'il s'arrête de se plaindre » $(1964,50,172)$. Mais, contrairement à l'héroïne de Duras, Nada ne recherchera pas à remettre en scène cette fascination. Plutôt, elle s'en défendra.

Depuis, elle s'est réfugiée dans ce que nous avions coutume d'appeler sa «grotte». Ce mot évoque bien sûr sa chambre dans laquelle elle s'est réfugiée à l'abri d'un monde qui lui fait peur, mais il fait aussi référence à la carapace qu'elle s'est constituée — un «moi-carapace» — surface dure et lisse que rien ne vient troubler. Loin des turbulences du corps. À l'abri des passions. Le lieu psychique lui-même devient ainsi le prolongement de ce lieu inviolable. Que tout s'immobilise, se pétrifie, afin que soit maintenu un espace interne à l'abri des effractions. «Je voudrais être une roche», me dit-elle parfois.

À partir des premiers écrits de Freud sur le féminin, Monique Schneider (2002, 70) propose une conception du féminin qui met l'accent sur l'opposition fermé/ ouvert plutôt que sur celle, controversée, de phallique/châtré. Le féminin, en effet, ne pourrait-il se concevoir que comme manque ou blessure?

«La femme n'est-elle pas exposée en première ligne à ce qui peut être rapporté à une irruption du vivant dans son propre 
espace, irruption venant ébranler les repères identitaires?» (Schneider, M., 2002, 72)

La pénétration n'est plus seulement celle du corps de la femme mais elle devient métaphore de l'intrusion d'un corps étranger dans le psychisme. Le refus du féminin serait dès lors protection farouche de cet espace intime - celui que Freud a désigné comme Hohlraum ou espace creux - refus de l'ouverture sur le dedans, sur l'inconscient.

Dès 1897, Freud avait esquissé cette correspondance entre le féminin et l'autre au-dedans de soi lorsqu'il avait écrit que «l'élément essentiel refoulé est toujours l'élément féminin » ou quand, des années plus tard, il fera du «refus du féminin » l'écueil majeur contre lequel se heurtera le processus analytique. Jacques André, dans Aux origines féminines de la sexualité, évoquant la passivité originaire de l'enfant devant la passion adulte, prolonge cette intuition freudienne en proposant une conjonction entre l'enfant séduit, effracté, et la position féminine.

«L'enfant séduit est un enfant cavité, un enfant orificiel [...]

L'être-effracté de l'enfant séduit anticipe et profile l'être pénétré de la féminité. » (André, J., 1995, 111)

Au début, l'Hilflosigkeit, cette «détresse originaire » du petit d'homme devant la passion adulte mais aussi face à l'excitation pulsionnelle vécue comme une agression par un corps étranger interne.

Nada ne peut investir son espace interne, celui-ci éveillant chez elle des angoisses d'intrusion-effraction tant corporelles que psychiques. Elle ne peut supporter de ressentir de «creux» au-dedans d'elle. Elle mange non pas parce qu'elle a faim mais pour ne pas sentir le vide de la faim. Dans la boulimie, écrit Jacques André (1995, 155), c'est à l'angoisse devant «l'ouverture féminine du corps, à l'immaîtrisable de celle-ci pour la psyché » que nous sommes confrontés. Ce que recherche Nada, c'est l'abolition de toute douleur et, avec elle, de tout émoi. «Vivre est trop difficile», me dira-t-elle. Dans ses rêves, des forces maléfiques envahissent la maison; il faut colmater les trous, les fissures par où l'angoisse pourrait s'infiltrer. Le danger, ce n'est pas seulement l'effraction, mais le fait qu'elle permette que s'installe, à l'intérieur d'elle, une vie étrangère, immaîtrisable.

Récemment, elle avait rêvé à moi. Nous étions dans sa chambre. «Voyez, c'est comme ça que je vis. » Elle se souvenait m'avoir dit cela et aussi d'avoir fermé la porte à clef pour que ses parents n'entrent pas. C'était la première fois qu'elle me laissait pénétrer dans son intimité. C'est ainsi qu'elle avait compris ce rêve. Elle en était à la fois troublée et heureuse. 
Elle avait souvent rêvé à moi depuis le début de l'analyse mais chaque fois, j'avais les traits d'une figure inquiétante, dotée de pouvoirs occultes ou possédant des appareils me permettant de voir l'intérieur de son corps. D'autre fois encore, j'étais la chirurgienne froide, inaccessible, la négociante retorse ou alors j'apparaissais sous les traits d'une étrangère dont il fallait se méfier. Toutes ces figures renvoyaient à un danger d'effraction de son enveloppe corporelle ou psychique dont il lui fallait se protéger à tout prix. Ce que Nada n'avait pu réaliser sur le plan psychique, elle avait tenté de le créer sur le plan du réel, de la vie matérielle, en se constituant un univers clos et protégé. Cette chambre où elle vivait, toujours la même, depuis le jour de sa naissance n'était que le prolongement du ventre maternel qu'elle n'arrivait pas à quitter.

Fermer la porte à clef, refuser l'entrée à ses parents, délimitant ainsi un espace en-dehors d'eux. C'était la première fois que, dans un rêve, nous nous retrouvions seules, elle et moi. La plupart du temps, l'espace de nos séances était toujours encombré de gens qui faisaient intrusion ou alors elles se déroulaient dans des lieux publics, sur le trottoir où il fallait marcher de plus en plus vite pour éviter que ses parents ne nous rattrapent. Je vis là une lueur d'espoir. Était-ce l'ébauche d'une séparation d'avec la mère, prélude à l'investissement de son propre espace interne ?

Nada n'a pas de pensée, pas de désir qui lui soient propres. Depuis toujours, elle s'est modelée sur ce que sa mère attendait d'elle. L'école et l'employeur ont pris le relais. Dans le transfert, cela s'exprime de diverses manières.

D'abord, par une attention soutenue, une vigilance extrême au moindre changement dans mon attitude, mon expression, entraînant chez elle soumission, ou alors fermeture contre ce qu'elle ressentira comme des tentatives d'instaurer un mouvement, de la ramener à la vie, ce contre quoi elle luttera durant des années. Se maintenir dans une identité de robot mécanique peut aussi se comprendre comme une tentative de stériliser le processus thérapeutique, de le faire avorter. C'est alors le sexuel dans la cure qui se refuse comme se refuse toute sexualité (la sienne mais aussi celle de la mère). Dès lors, l'analyse ne peut être féconde. Réussirai-je, moi, la chirurgienne, là où la mère avait échoué?

À d'autres moments, elle aurait voulu que je sois plus brutale, plus confrontante. Il lui semblait que seul un choc violent venant de l'extérieur pourrait la sortir de sa torpeur. Elle-même se sentait paralysée, soumise à une force d'inertie qui l'empêchait de s'animer. Elle s'imaginait alors comme une surface vierge (Etch-a-sketch $\left.{ }^{2}\right)$ sur laquelle je dessinerais pour elle le tracé de sa vie. Ou alors, elle se visualisait comme une de ces boules de verre qu'on trouve à Noël, avec à l'intérieur un paysage enneigé. Elle me demandait alors de la secouer. Chaque fois que j'y succomberai, en me faisant plus active, le voile de l'oubli recouvrira tout jusqu'à la séance suivante et réduira mes efforts à néant. Ou alors, elle dormira pendant des jours, s'anesthésiant dans un sommeil sans rêves, dans lequel elle se réfugiera.

Dans le sommeil, espace narcissique et espace maternel se rejoignent. Dans L'inquiétante étrangeté, Freud souligne que le fantasme d'être enterré vivant «n'est que la transmutation d'un autre [fantasme] rempli d'une certaine volupté, à 
savoir le fantasme de vivre dans le sein maternel.» (1919, 250) Dormir pour retourner dans le ventre maternel mais aussi pour retrouver un état d'avant la naissance, d'avant la vie. C'est aussi le principe de Nirvâna (le retour de l'excitation au niveau zéro) élaboré par Freud dans Au-delà du principe de plaisir.

Dans cette chambre où elle vit, où elle dort, prend tous ses repas, Nada entasse tout ce qu'elle possède, des boîtes, entre autres, qu'elle aime collectionner, des boîtes décoratives, par groupe de trois, qui s'emboîtent les unes dans les autres à la manière de poupées gigognes. Qu'y met-elle ? Rien. Elle les achète, me dit-elle, parce qu'elle les trouve belles. Tout simplement. Elle n'en a aucun besoin. Mais elle ne peut s'empêcher d'en acheter.

«Si nous avions affaire à un rêve, nous penserions aussitôt que les coffrets sont aussi des femmes, des symboles de ce qui est l'essentiel en la femme.» (Freud, 1913) [Nous sommes loin de la théorie phallocentrique à laquelle il s'accrochera plus tard]

Mais, au-delà, qu'est-ce que Nada me donne à voir par toutes ces boîtes qui encombrent sa chambre? Des boîtes, comme des ventres dans lesquels elle mettrait des bébés-boîtes? Mais alors, à quoi renvoie ce vide ? À l'automate, vide de toute substance? Cette pureté du vide auquel Nada aspire, serait-ce le reflet de celui qui avait habité sa mère lorsqu'elle était enceinte d'elle? Qu'aurait-elle été alors sinon qu'un vide à l'intérieur de sa mère ? Peut-être est-ce ce qu'elle cherche à me dire lorsqu'elle me répète: je ne suis Rien. Récemment, en repensant à ces boîtes, elle m'a avoué que c'était pour cacher tout ce qui n'était pas beau en elle ; le mal, la honte au-dedans d'elle.

Jusqu'à présent, Nada a échoué à vivre le féminin, à investir cet espace «creux », «espace du maternel primaire», comme l'a élaboré Florence Guignard (2007), espace intérieur, source de vie et de créativité. Elle a échoué comme on pourrait dire aussi d'une épave rejetée par la mer, puis oubliée. Carcasse vide de toute vie. Peut-être est-ce là, ce qu'il y a derrière cet attachement «fou » à la mère, une mère dont elle ne peut se séparer?

«Je ne peux vivre sans elle», me répète-t-elle.

Un jour, en venant à sa séance, elle avait marché comme elle le faisait toujours sur la rue qui longeait le fleuve. Elle avait eu cette image qui s'était imposée à elle : des petits canards de bois comme ceux que l'on voit dans les stands de tir à La Ronde. Elle s'était imaginé être l'un deux, passant et repassant devant l'ouverture - elle faisait le mouvement en même temps qu'elle m'expliquait — jusqu'à ce qu'il arrive au bout et Plouf !, il disparaît. C'est à ce moment qu'elle avait pensé se jeter dans les eaux glacées du fleuve. Mais, pas maintenant. Pas tant que sa mère vivait. Elle aurait trop de peine. 
Elle aurait souhaité que sa mère l'encourage dans ses tentatives d'individuation mais, chaque fois qu'elle lui parlait de partir en appartement, sa mère lui répondait: «Reste avec moi. Tu n'es pas bien ici ?» ou alors «Attends que je meure; je n'en ai pas pour longtemps. » Cette supplique maternelle durait depuis plus de dix ans.

Ainsi se dessine l'emprise de la mère sur la fille. Ainsi s'énonce le pacte qui les lie: Je ne peux vivre sans toi. Tu ne peux vivre sans moi. Mais, qu'y a-t-il derrière cet amour terrible qui lie la fille à sa mère, l'assujettit à elle, sinon une quête éperdue et toujours impossible pour l'amour de la mère. «Tu n'as jamais été, tu ne seras jamais, la cause de mon désir» (Pontalis, 1988, 123), telle est, au fond, la blessure que recouvre cet attachement désespéré à la mère.

Dans La lettre et la mort (2004, André Green, évoquant le fantasme de ne plus avoir de corps, avance qu'il y a une correspondance entre l'absence de corps et l'absence de temps. Cela m'a aidée à comprendre la relation particulière qu'entretient Nada avec le temps. Ne pas bouger, ne rien changer, c'est aussi pour Nada une tentative pour suspendre le temps. Ne pas vieillir, que l'usure du temps ne l'atteigne pas, ni elle, ni ses parents. Sa mère, surtout, sans laquelle toute vie lui apparaît impossible. Nous sommes ainsi dans une répétition qui refuse la vie, non pas simple stagnation mais ce qu'André Green appelle le «meurtre du temps». Tuer le temps, n'est-ce pas le meurtre accompli par la mère lorsqu'elle ne peut accepter que sa fille devienne femme et qu'elle en vienne à se détacher d'elle pour vivre «sa » vie? Nada demeure ainsi prisonnière du lien à sa mère, attendant d'elle une autorisation à vivre sa vie qui ne vient jamais.

Faire le vide. Dormir. N'est-ce pas pour Nada la seule façon de ne pas trahir cette mère en la quittant? Risquer de la perdre, c'est aussi risquer pour elle de tout perdre, de se perdre. Devenir une roche, une automate sans vie, construire une digue contre le danger d'être submergée par la vague pulsionnelle, mais sans doute aussi contre la vague maternelle. Une mère qui avait échoué à être femme, à être mère de sa fille.

Double échec. D’une part, dans la création de ce que Florence Guignard (2007) a appelé l'espace du «maternel primaire», lieu des premiers investissements pulsionnels, de l'intériorisation de la capacité de «rêverie» maternelle. D'autre part, échec à constituer l'espace du «féminin primaire», lieu des premières triangulations, de l'identification au désir de la mère pour le père.

«L'écriture, a dit Duras, est la seule chose qui soit plus forte que ma mère.» Dans son livre Un barrage contre le Pacifique (Duras, 1950), elle nous montre la folie d'une mère qui se bat contre l'inexorable montée des eaux qu'elle tentera en vain d'endiguer. Si l'écriture, pour Duras, fit barrage contre cet emportement maternel, en créant un espace insaisissable, est-ce que la thérapie permettra à Nada de créer ce même espace? 
Puis, il y eut des changements. Une parole plus spontanée, moins soucieuse du mot juste, de l'intelligence du propos. Des pleurs, des flots de larmes qu'elle laissait couler devant moi de plus en plus souvent. Son corps reprenait vie tranquillement. Elle émergeait de ce qu'elle appelait son ère glaciaire. Mais, en même temps, la mécanique se déréglait. Il y a quelques mois, des douleurs apparurent aux bras. Les médecins diagnostiquèrent des tendinites, des déchirures dans les tendons. On lui signa des papiers l'exemptant de tout travail manuel. Ce qu'elle avait tenté d'évacuer psychiquement faisait maintenant retour par le corps. Elle repensait à son rêve où elle se cramponnait à la plate-forme pour ne pas tomber dans le vide. Peut-être avait-elle développé des tendinites à force de se cramponner, de s'agripper? Y avait-il quelque chose en elle, inconnu d'elle-même, qui agissait à son insu ? Cela la terrifiait de penser cela.

«Le crépitement du vivant », c'est ainsi qu'elle nommait à présent ses tensions aux bras, aux jambes, les sursauts qui agitaient son corps par moments, ses élans de «folie», les mots qui sortaient de sa bouche sans censure. «Peut-être est-ce ainsi parce que ça a été pétrifié, gelé, trop longtemps?» me dit-elle. Elle avait pensé à une pièce de viande qui dégèle sur le comptoir. Elle avait évoqué sa fascination pour les cadavres. Entre autres, elle raffolait des séries télévisées qui montraient des autopsies, où l'on pouvait voir avec beaucoup de réalisme le processus de décomposition à l'intérieur du corps. Elle qui rêvait de mécaniques, d'automates aux corps vides de toute substance, était étonnée qu'il puisse y avoir «tant de vie même dans un cadavre».

Elle devenait de plus en plus sensible. À une infirmière qui lui avait dit: «Ce n'est pas grave, ça s'opère.», elle avait répondu, révoltée: «Je ne suis pas une machine qu'on répare.» De plus en plus, elle ressentait de la colère contre l'employeur qui l'exploitait, contre son père par qui elle s'était toujours sentie méprisée. D' autre part, elle ne pouvait toujours pas s'imaginer quitter l'usine sans que l'angoisse ne l'étreigne, sans avoir l'impression de se lancer dans le vide. Partir mais pour aller où ? Un médecin lui avait dit qu'elle devrait faire travailler sa tête, sa cabesa, mais en était-elle seulement capable? Elle voudrait que je la programme. Le « roc» contre lequel se heurtera l'analyse, avait dit Freud, c'est le refus du féminin pour les deux sexes, refus qui peut s'entendre comme le refus de l'ouverture sur le dedans, sur l'inconscient, comme protection farouche d'un espace intime. Dans le mouvement même de toute cure, il y a une nécessaire passivité, ouverture et soumission à l'empreinte de l'autre. C'est toute la difficulté avec des patients comme Nada pour qui cette passivité répète une passivité originaire dans un lien qui se veut aliénant. «Programmez-moi !», me demande-t-elle. Mais répondre à une telle demande nous conduirait à coup sûr à une impasse car, pour naître à la vie psychique, il lui faut se défendre contre les empiètements de l'objet - aussi bien ceux de l'analyste/thérapeute que ceux de la mère. 
Nada était venue me voir pour que je la guérisse de sa sexualité, pour que je la débarrasse de ses «tensions » sexuelles.

«Le vagin est la chose même, a dit Jacques André, parce qu'il est le lieu répétitif de l'intrusion séductrice originaire. Comme lieu de pénétration, le vagin est propre à ressaisir, à symboliser, l'intromission de la sexualité adulte dans le psychosoma de l'enfant. » (André, J., 1995, 130) ${ }^{3}$

Était-ce ce que Nada avait cherché à me dire par ces contractions dont son corps était le théâtre impuissant? Que tentaient-elles de symboliser? De quelles effractions-pénétrations portaient-elles la trace?

Dernièrement, alors qu'elle commençait à se sentir plus vivante, ses «tensions sexuelles » réapparurent. Elle prit peur. Pendant les jours qui ont suivi, elle s'enferma dans sa routine. À nouveau, elle sentait ce qu'elle appelait elle-même sa force d'inertie la recouvrir comme une chape de plomb. Elle avait «les bras morts », la «tête vide».

«Et si la mort ne s'arrêtait pas là, si elle montait le long de ses bras jusqu'à sa colonne vertébrale?», me confia-t-elle. Elle avait pensé à la sclérose en plaques.

«Guérir de la sexualité, guérir de l'excès de mère, ne pas vouloir guérir, c'est tout un. » a écrit Pontalis (1988, 115). À l'horizon, on retrouve toujours la mère, pas tant la mère réelle, que la mère comme objet interne, celle dont Nada espère encore l'amour. Refuser tout changement, serait-ce alors refuser de trahir la mère et garder l'espoir de la faire fléchir?

«Plutôt rester malade que tomber guérie.» J'emprunte à Pontalis $(1988,93)$ cette formulation qui s'applique très bien à Nada. Car guérir, c'est rencontrer la souffrance et, avec elle, l'inévitabilité de la perte.

suzanne tremblay

620 notre-dame

saint-lambert

j4p 211

suzan.tremblay@videotron.qc.ca

\section{Notes}

1. Le présent texte a fait l'objet d'une présentation en avril 2007 lors du colloque de l'APPQ, Être au féminin.

2. Référence à un jouet, sorte d'écran sur lequel on dessine des tracés à l'aide d'un aimant et qu'on peut effacer en le secouant; contrairement au bloc-notes magique de Freud, les dessins successifs ne laissaient pas de traces visibles.

3. On pense bien sûr aussi à S. Ferenczi et à La confusion des langues, au signifiant énigmatique de J. Laplanche. 


\section{Bibliographie}

André, J., 1995, Aux origines féminines de la sexualité, PUF.

Duras, M., 1950, Un barrage contre le Pacifique, Gallimard.

Duras, M., 1964, Le ravissement de Lol. V. Stein, Gallimard.

Fedida, P., 1978, L'absence, NRF, Gallimard.

Freud, S., 1897, La naissance de la psychanalyse, PUF, 1956.

Freud, S. 1913, Le motif du choix des coffrets, in L'inquiétante étrangeté et autres essais. Gallimard, 1985.

Freud, S., 1919, L'inquiétante étrangeté,, in L'inquiétante étrangeté et autres essais. Gallimard, 1985.

Freud, S.,1920, Au-delà du principe de plaisir, in Essais de psychanalyse. Payot, 1981.

Green, A., 2004, La lettre et la mort : promenade d'un psychanalyste à travers la littérature. Denoël.

Guignard, F., 2007, Le Maternel et le Féminin: Deux espaces de la vie psychique. Communication présentée à l'APPQ, avril 2007.

Pontalis, J.B., Non, deux fois non, in Perdre de vue, Gallimard, 1988.

Schneider, M., 2002, Freud et le rapport féminin à la négation, in Fatalités du féminin, PUF. 